\title{
IMAGING LOCAL SCATTERER CONCENTRATIONS BY THE NAKAGAMI STATISTICAL MODEL
}

\author{
Po-Hsiang Tsui and Chien-Cheng Chang \\ Institute of Applied Mechanics, National Taiwan University, Taipei 106, Taiwan and Division of Mechanics, \\ Research Center for Applied Sciences, Academia Sinica, Taipei 115, Taiwan
}

(Received 9 June 2006; revised 29 September 2006; in final form 10 October 2006)

\begin{abstract}
The ultrasonic B-mode image is an important clinical tool used to examine the internal structures of the biological tissue. Due to the fact that the conventional B-scans cannot fully reflect the nature of the tissue, some useful quantitative parameters have been applied to quantify the properties of the tissue. Among various possibilities, the Nakagami parameter was demonstrated to have an outstanding ability to detect the variation of the scatterer concentration. This study is aimed to develop a scatterer concentration image based on the Nakagami parameter map to assist in the B-mode image for tissue characterization. In particular, computer simulations are carried out to generate phantoms of different scatterer concentrations and echogenicity coefficients and their B-mode and Nakagami parametric images are compared to evaluate the performance of the Nakagami image in differentiating the properties of the scatterers. The simulated results show that the B-mode image would be affected by the system settings and user operations, whereas the Nakagami parametric image provides a comparatively consistent image result when different diagnosticians use different dynamic ranges and system gains. This is largely because the Nakagami image formation is only based on the backscattered statistics of the ultrasonic signals in local tissues. Such an imaging principle allows the Nakagami image to quantify the local scatterer concentrations in the tissue and to extract the backscattering information from the regions of the weaker echoes that may be lost in the B-mode image. These findings suggest that the Nakagami image can be combined with the use of the B-mode image simultaneously to visualize the tissue structures and the scatterer properties for a better medical diagnosis. (E-mail: mechang@gate.sinica.edu.tw) (c) 2007 World Federation for Ultrasound in Medicine \& Biology.
\end{abstract}

Key Words: Nakagami distribution, Parametric image, Ultrasonic backscattering.

\section{INTRODUCTION}

The gray-scale ultrasound image system (B-mode) is a frequently-used tool noninvasively to examine the tissue anatomy in the medical diagnosis. The gray-scales in the B-mode image are determined according to the strengths of the echoes from the changes in acoustic impedance in the tissues. However, we should note that the use of the B-mode image in the clinical diagnosis might suffer from some disadvantages, described as follows.

First, more clearly to visualize the structures in a tissue, the typical ultrasonic scanner allows operators to adjust different system parameters, such as the system gain, time-gain compensation (TGC) and dynamic range. Moreover, different ultrasonic scanners made by differ-

Address correspondence to: Chien-Cheng Chang, Division of Mechanics, Research Center for Applied Sciences, Academia Sinica, 128, Section 2, Academia Road, Nankang, Taipei 11529, Taiwan. E-mail: mechang@gate.sinica.edu.tw ent manufacturers have different procedures of signal and image processing. It means that the B-mode image is easily affected by the system factors. Second, the specular reflection is highly angle-dependent (Shung and Thieme 1993). When the sound beam is perpendicular to the interface of the tissue, the transducer can receive a large amount of the returned acoustic energy. Otherwise, only less energy is received. It implies that the brightness of the B-mode image also relies on the skill and training of the operators. Third, during imaging, the scattering will occur when the incident wavelength is greater that or comparable with the dimension of the scatterers in a tissue. The generated backscattered signals would form the so-called speckle, which often exhibits a granular pattern of white and dark spots in the ultrasonic B-mode image (Shung et al. 1992). To avoid the influence of the speckle effect on the image quality, many methods were proposed to reduce the speckle appearance in the Bmode image (Burckhardt 1978; Berson et al. 1981; Tra- 
Table 1. The scatterer properties of the type-II phantoms, where $c_{r}$ is the relative echogenicity coefficient

\begin{tabular}{lccc}
\hline & $\begin{array}{c}\text { Scatterer } \\
\text { concentration } \\
\text { in the } \\
\text { background } \\
\left(\mathrm{mm}^{-2}\right)\end{array}$ & $\begin{array}{c}\text { Scatterer } \\
\text { concentration } \\
\text { in the } \\
\text { cylinder } \\
\left(\mathrm{mm}^{-2}\right)\end{array}$ & $\begin{array}{c}c_{r} \text { (background) } \\
: c_{r} \text { (cylinder) }\end{array}$ \\
\hline Phantom no. 1 & 16 & 16 & $5: 1$ \\
Phantom no. 2 & 16 & 6 & $1: 5$ \\
Phantom no. 3 & 6 & 16 & $5: 1$ \\
\hline
\end{tabular}

hey et al. 1986). Nevertheless, due to the fact that the backscattered signals are actually dependent on the shape, size, density and other properties of the scatterers in a tissue (Bamber and Hill 1981; Bamber et al. 1981; Thijssen 1989; Insana et al. 1990), the information related to the scatterers carried by both the backscattered echoes and other weak signals might be lost in the B-mode image.

To resolve the dilemmas of the ultrasonic B-scans, different kinds of quantitative methods that are independent of the effects from the systems and operators are developed to provide the information of the scatterers, which may be associated with the nature of the biological tissues, for assisting in the diagnosis of the B-mode image. Considering the randomness of the ultrasonic backscattered signals, many researchers apply various statistical distributions to model the shape of the probability density function (pdf) of the backscattered echoes for the tissue characterization. The Rayleigh distribution is the first model used to describe the statistics of the ultrasonic backscattered signals (Burckhardt 1978). The pdf of the backscattered envelope would follow the Rayleigh distribution when the resolution cell of the ultrasonic transducer contains a large number of randomlydistributed scatterers. It should be noted that the scatterers in most biological tissues have various possibilities of arrangements. If the resolution cell contains the scatterers that have randomly varying scattering cross-sections with a comparatively high degree of variance, the envelope statistics are the pre-Rayleigh distributions. If the resolution cell contains the periodically-located scatterers in addition to the randomly-distributed scatterers, the envelope statistics are the post-Rayleigh distributions. And therefore some useful distributions including Rician (Wagner et al. 1987), K (Weng et al. 1991), homodyned K (Dutt and Greenleaf 1994) and generalized K (Shankar 1995) are applied to encompass both the pre-Rayleigh and post-Rayleigh statistics of the backscattered envelope. However, the computational complexity of these models in their parameter estimations may limit their practical applications.
Several years ago, the Nakagami distribution, initially proposed to describe the statistics of the radar echoes, was applied to the statistical analysis of the ultrasonic backscattered signals (Hampshire et al. 1988; Zimmer et al. 1996; Shankar 2000; Wachowiak et al. 2002). The Nakagami statistical model has comparatively less computational complexity and is general enough to describe a wide range of the scattering conditions in medical ultrasound, including pre-Rayleigh, Rayleigh and post-Rayleigh distributions. Although the Nakagami distribution can fit well with the pdf of the ultrasonic envelope, a compounding statistical distribution may be more appropriate to model the envelope statistics, because the ultrasonic signals returned from the tissues may contain contributions from more than one mechanism. Hence, the compounding Nakagami distributions involving the Nakagami-gamma (Shankar 2003; Shankar 2004), Nakagami-lognormal (Karmeshu and Agrawal 2006), and Nakagami-inverse Gaussian (Karmeshu and Agrawal 2006) were subsequently developed to fit more closely to the envelope statistics of the ultrasonic backscattered echoes from tissues. In these distributions, the primary parameter to determine the backscattered statistics is the Nakagami parameter, which is estimated from the statistical moments of the ultrasonic backscattered envelope. It only depends on the shape of the backscattered envelope and has been demonstrated using computer simulations and experiments on phantoms (Shankar 2000; Tsui and Wang 2004) to have a good ability to differentiate different scatterer concentrations in a medium. The Nakagami parameter has also been applied to practical measurements on biological tissues to classify the scatterer properties, such as the bone (Wang and Tsai 2001), skin (Raju and Srinivasan 2002), breast (Shankar et al. 2001; Shankar et al. 2003b) and blood (Cloutier et al. 2004).

As a result of the outstanding ability of the Nakagami parameter to detect the variation of the scatterer concentration, this parameter was suggested to form the parametric image in making a medicine diagnosis (Shankar 2002) and, afterward, some preliminary studies and applications have been performed (Kolár et al. 2004; Davignon et al. 2005). However, there is no complete report related to the Nakagami parametric image to date. This study is aimed to develop a local scatterer concentration image based on the Nakagami parameter map. In particular, computer simulations are carried out to generate phantoms with different concentrations and echogenicity coefficients of the scatterers. On the one hand, the B-mode images under various system gains and dynamic ranges are generated. On the other hand, an optimal size of the window that satisfies the stable estimation 
(a)
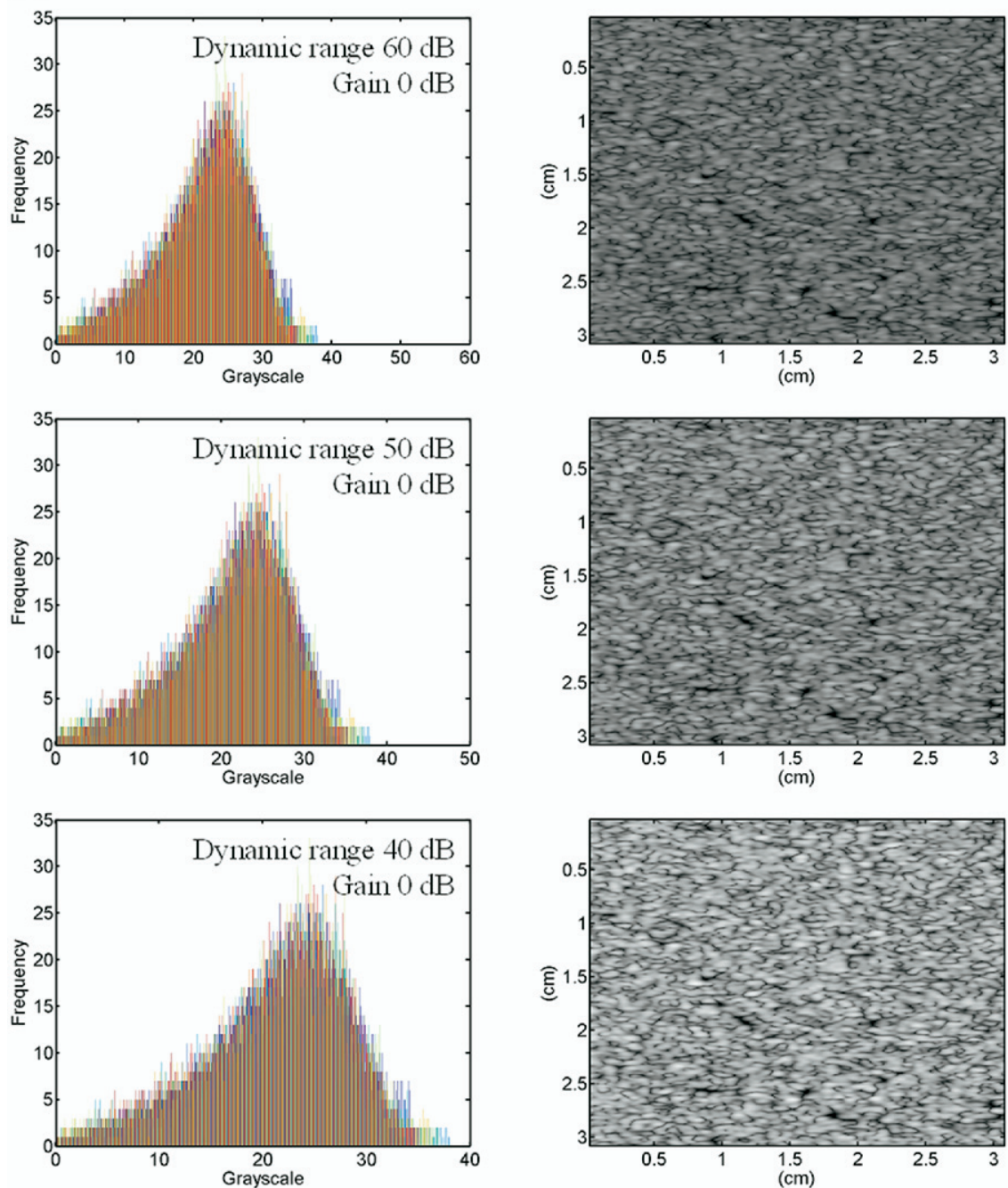

Fig. 1. The B-mode image and its gray-scale histogram for the type-I phantom of the scatterer concentration of 8 scatterers $/ \mathrm{mm}^{2}$. (a) Constant system gain and different dynamic ranges; (b) Constant dynamic range and different system gains.

of the local Nakagami parameter is determined for constructing the Nakagami images of the phantoms. The B-mode and Nakagami images are compared to evaluate the performance of the Nakagami image in quantifying the scatterer properties in biological tissues. Finally, we will discuss and remark on the possible role and contributions of the Nakagami image in the medical techniques.

\section{THEORETICAL BACKGROUND AND METHODS}

\section{Nakagami distribution}

The pdf $f(r)$ of the ultrasonic backscattered envelope $R$ under the Nakagami statistical model is given by

$$
f(r)=\frac{2 m^{m} r^{2 m-1}}{\Gamma(m) \Omega^{m}} \exp \left(-\frac{m}{\Omega} r^{2}\right) U(r)
$$

where $\Gamma(\cdot)$ and $\mathrm{U}(\cdot)$ are the gamma function and the unit step function, respectively. Let $E(\cdot)$ denote the statistical mean, the scaling parameter $\Omega$ and the Nakagami parameter $m$ associated with the Nakagami distribution can be, respectively, obtained from

$$
\Omega=E\left(R^{2}\right)
$$

and

$$
m=\frac{\left[E\left(R^{2}\right)\right]^{2}}{E\left[R^{2}-E\left(R^{2}\right)\right]^{2}} .
$$

The Nakagami parameter $m$ is a shape parameter to 
(b)
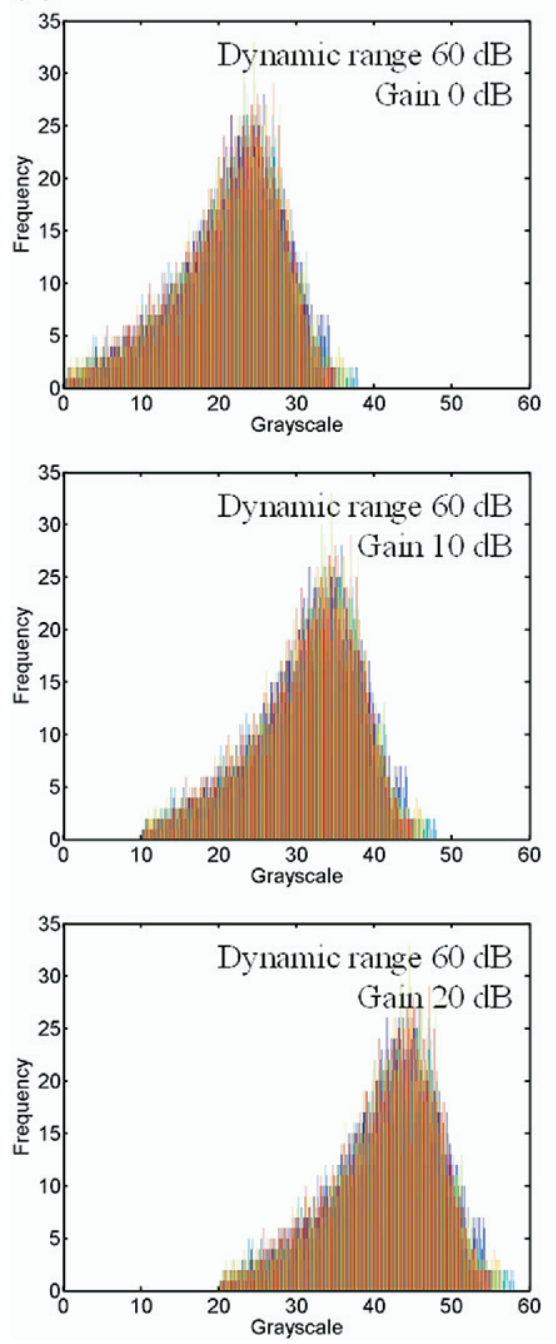
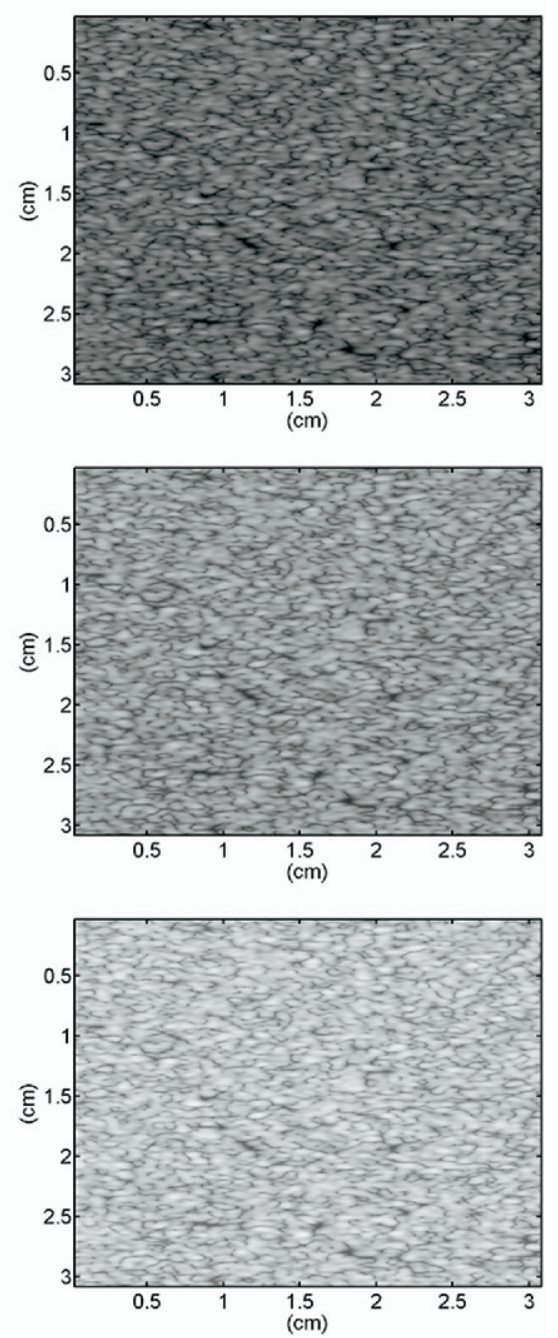

Fig. 1. Continued.

determine the statistical distribution of the ultrasonic backscattered envelope. As the parameter $m$ varies from 0 to 1 , the statistics of the envelope changes from preRayleigh to the Rayleigh distribution and the backscattered statistics is post-Rayleigh distribution if $m$ is larger than 1 . Because different scatterer concentrations result in different envelope statistics, the parameter $m$ is a good parameter for quantifying concentrations of scatterers in the tissues.

\section{METHODS}

We apply the system-based model (Bamber and Dickinson 1980; Meunier and Bertrand 1995; Fontaine et al. 1999; Fontaine et al. 2002; Teh and Cloutier 2000; Tsui and Wang 2004) to simulate the ultrasonic backscattered echoes and the B-mode image of the biological tissue. Firstly, to reduce the computational complexity, the 3-D model is simplified to a model in two dimensions. The acoustic attenuation, beam diffraction and system noise are not considered to achieve the ideal and undistorted shape of the ultrasonic backscattered signals. Subsequently, the computer phantoms with different scatterer concentrations are constructed, in which the sound velocity is $1540 \mathrm{~m} / \mathrm{s}$ and the internal scatterers are assumed to be randomly distributed point targets with the same relative echogenicity coefficient $c_{\mathrm{r}}$. For the convenient discussion, we call these phantoms the type-I phantom. Then, a Gaussian incident wave with a central frequency of $5 \mathrm{MHz}$ is generated, in which the $-6 \mathrm{~dB}$ bandwidth is $4 \mathrm{MHz}$ and the pulse length and the $-3 \mathrm{~dB}$ beamwidth are $0.9 \mathrm{~mm}$ and $0.5 \mathrm{~mm}$, respectively. Consequently, the simulated ultrasonic backscattered signals 
(a)
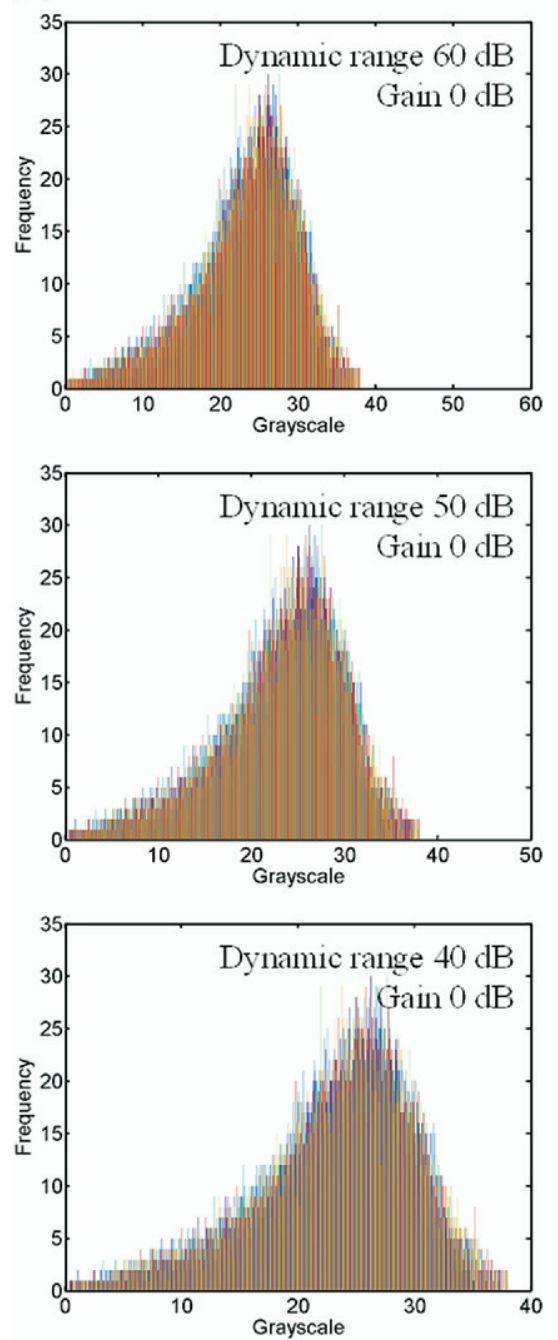
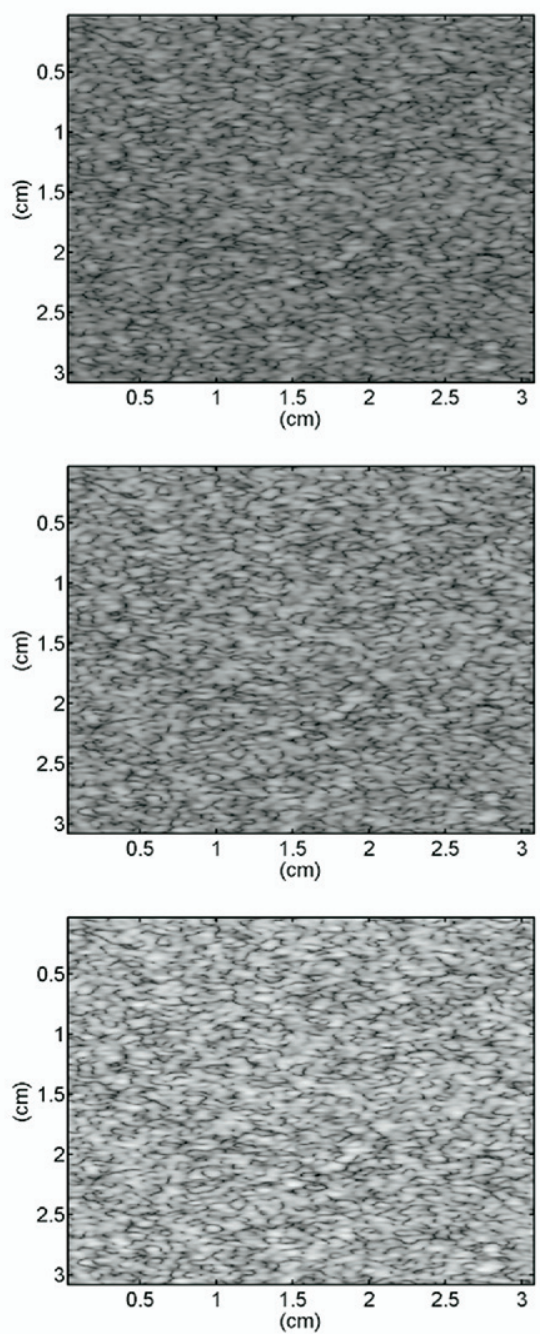

Fig. 2. The B-mode image and its gray-scale histogram for the type-I phantom of the scatterer concentration of $16 \mathrm{scatterers} / \mathrm{mm}^{2}$.

(a) Constant system gain and different dynamic ranges; (b) Constant dynamic range and different system gains.

(scan-lines for imaging) correspond to the convolution of the incident wave with the computer phantom. For each phantom of a specific scatterer concentration, an area consisting of totally 1000 image scan-lines corresponding to approximately $3 \times 3 \mathrm{~cm}^{2}$ is selected as the region-of-interest (ROI) for imaging and analysis. Each scan-line is demodulated to obtain the envelope image of the ROI and the B-mode images of different system gains and dynamic ranges are formed from the logarithm-compressed envelope image.

The Nakagami parametric images for the phantoms of different scatterer concentrations are generated using the sliding window to process their envelope images. The sliding window technique is a typical method to form the ultrasonic parametric image (Valckx et al. 2000) and mainly contains two steps. (1) A subimage, termed a window, within the envelope image of the ROI is chosen to collect the local backscattered envelopes for estimating the local Nakagami parameter $m_{w}$. The parameter $m_{w}$ is then assigned as the new pixel located in the center of the window. (2) Let the window move throughout the whole envelope image in steps of one pixel and repeat the step 1. Thus, the Nakagami image of the ROI can be constructed using the map of the parameter $m_{w}$.

Note that the window size determines the resolution of the ultrasonic parametric image. As the window size decreases, the resolution of the parametric image gets better. However, the small window has less numbers of the envelope data, leading to an unstable estimation of the parameter $m_{w}$. Therefore, before the construction of the Nakagami image, the optimal size of the window that can simultaneously satisfy both the stable estimation of $m_{w}$ and an acceptable resolution of the parametric image needs to be determined. The procedure which we use to 
(b)
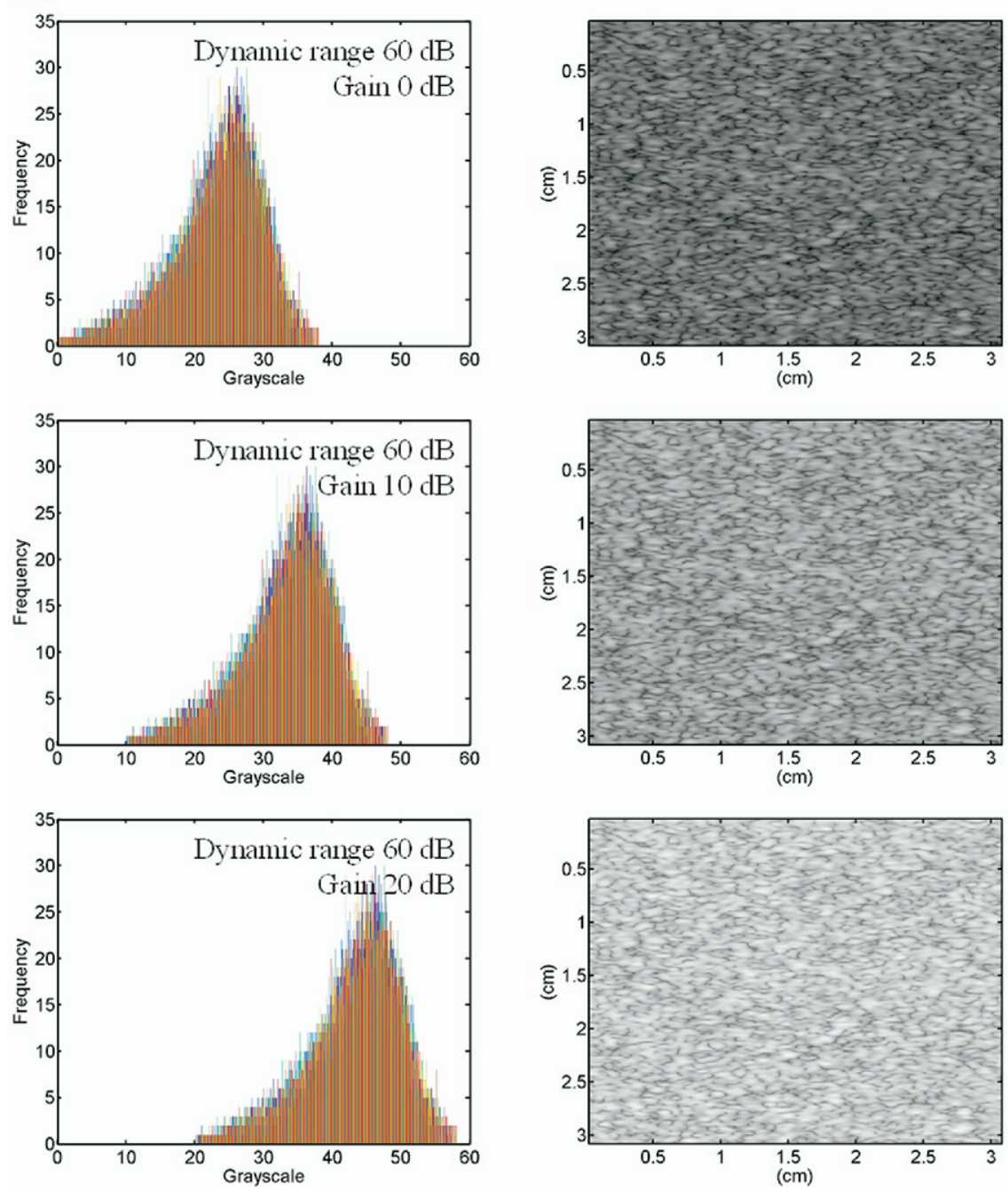

Fig. 2. Continued.

determine the optimal window size is described as follows.

(1) For the ROI, each envelope signal of the image scan-line is used to calculate the Nakagami parameter $m$ and the average Nakagami parameter $\bar{m}$. Compared with the previous studies, a 3-cm-long envelope signal of the image scan-line in the ROI is certainly large enough to satisfy the stable estimation of $m$, and $\bar{m}$ can be treated as an indicator of the global backscattered statistics of the ROI.

(2) Each $m_{w}$ in the ROI and their average value $\bar{m}_{w}$ is subsequently estimated using different increasing sizes of the sliding window. Assuming that the window size is large enough (but not too large) to satisfy the stable estimation of the parameter $m_{w}$, the result of $\bar{m}_{w}$ should approach that of $\bar{m}$ to reflect the identical global backscattered statistics of the ROI. Con- sequently, the optimal window size is determined once $\bar{m}_{w}=\bar{m}$.

After determination of the window size, the Nakagami images of those type-I phantoms are constructed. With the same simulation procedure and the same size of the sliding window, we further explore and compare the B-mode and the Nakagami-mode images of another three phantoms. In contrast to the type-I phantom, we classify these phantoms as the type-II phantom. They consist of a background material containing a cylinder object with a diameter of $1 \mathrm{~cm}$ and have different $c_{\mathrm{r}}$ and scatterer concentrations in the background and in the embedded cylinder. The scatterer properties of these type-II phantoms are shown in Table 1.

In order clearly to reveal the information of the Nakagami parametric image, both the gray-scale and pseudocolor are applied to display the Nakagami parameter map. 


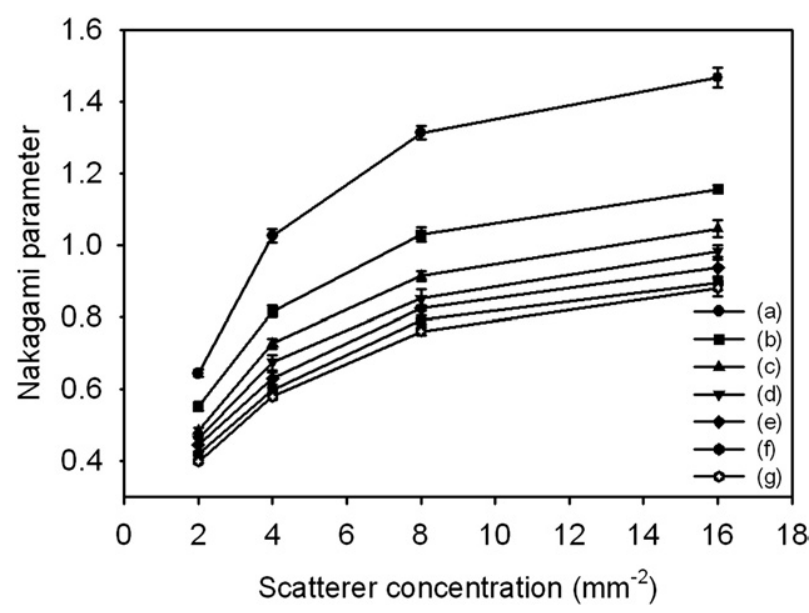

Fig. 3. The curves of $\bar{m}_{w}$ as a function of scatterer concentration estimated using different window sizes. (a) $0.9 \times 0.9 \mathrm{~mm}^{2}$; (b) $1.35 \times 1.35 \mathrm{~mm}^{2}$; (c) $1.8 \times 1.8 \mathrm{~mm}^{2}$; (d) $2.25 \times 2.25 \mathrm{~mm}^{2}$; (e) $2.7 \times 2.7 \mathrm{~mm}^{2}$; (f) $3.15 \times 3.15 \mathrm{~mm}^{2}$. (g) The curve of $\bar{m}$ as a function of scatterer concentration estimated from the envelope signal of the image scan-line. The simulations are repeated five times in order to obtain the mean value and the standard deviation.

The parameters $m_{w}$ smaller than 1 are assigned the blue shades, varying from deep to shallow with the increasing parameter values, representing various pre-Rayleigh statistics of the backscattered envelope. When $m_{w}$ equal 1 , the shade is white in order to express the Rayleigh distribution and those larger than 1 are assigned the shades of progressive red color in accordance with the increasing parameter value, indicating the incremental degree of the post-Rayleigh distributions in the backscattered statistics.

\section{RESULTS AND DISCUSSION}

The results obtained from the computer simulations are divided into four parts for the discussion. The Bmode images of the type-I phantoms with different scatterer concentrations are discussed first. Then, the optimal window size in the formation of the Nakagami image and the Nakagami images of the type-I phantoms are determined. Finally, the B-mode and Nakagami images of the three type-II phantoms (no. 1 to 3 ) are compared.

\section{The B-mode images of the type-I phantom}

Figure 1 shows the B-mode images and the corresponding gray-scale histograms under different dynamic ranges and system gains for the type-I phantom with a scatterer concentration of 8 scatterers $/ \mathrm{mm}^{2}$. The histogram of the image gray-scale broadens with decreasing the dynamic range of the B-mode image from 60 to 40 $\mathrm{dB}$ when the system gain is constant. This enhances the image contrast without changing the mean echo amplitude. If we fix the dynamic range at $60 \mathrm{~dB}$ as the system gain is increased from 0 to $20 \mathrm{~dB}$, the histogram of the image gray-scale maintains the same width and shape but shifts toward the right side, increasing the overall bright-
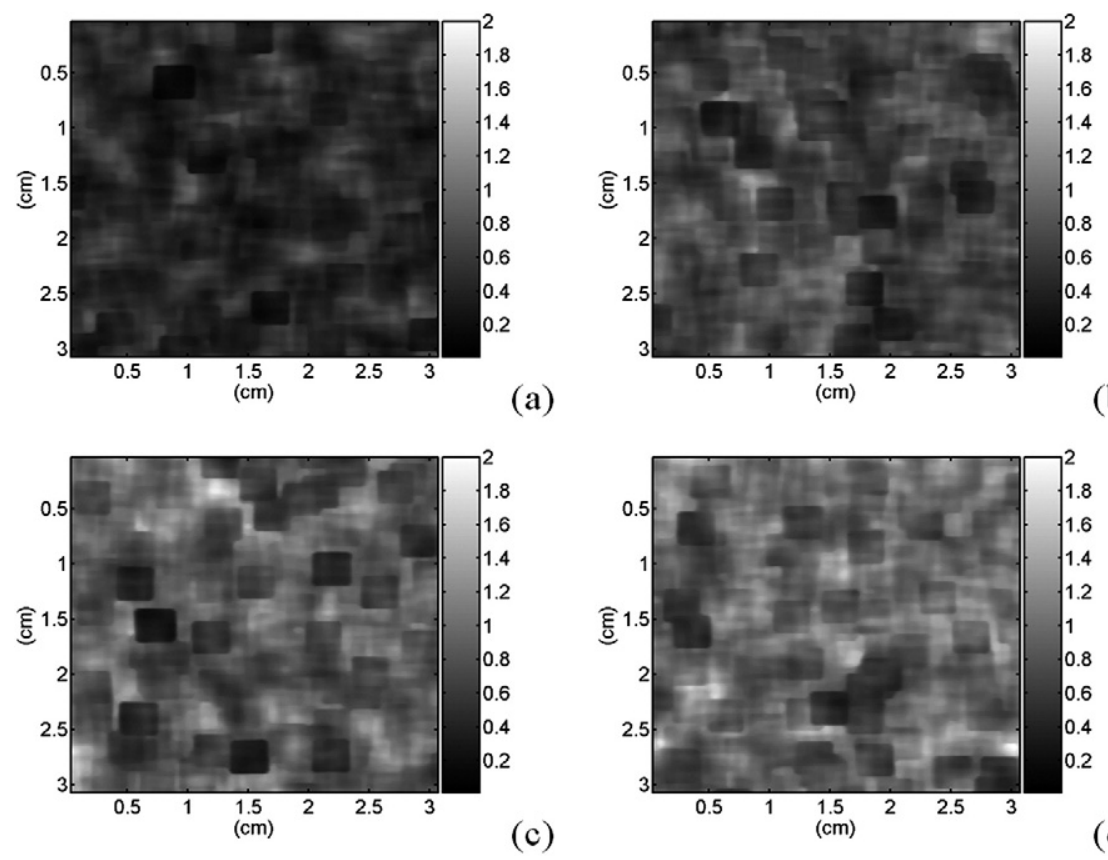

(b)

Fig. 4. The Nakagami parametric images with a gray-scale display for the type-I phantoms of various scatterer concentrations. (a) 2 scatterers $/ \mathrm{mm}^{2}$; (b) 4 scatterers $/ \mathrm{mm}^{2}$; (c) 8 scatterers $/ \mathrm{mm}^{2}$; (d) $16 \mathrm{scatterers} / \mathrm{mm}^{2}$. 

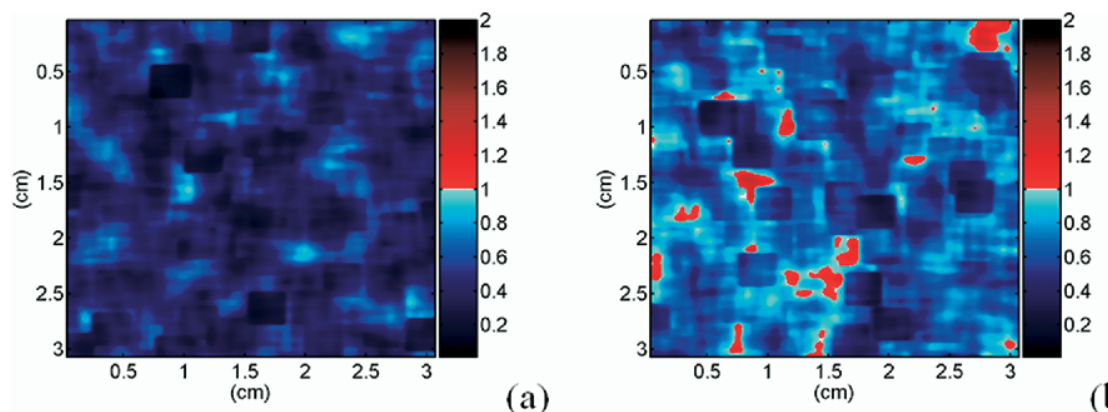

(a)
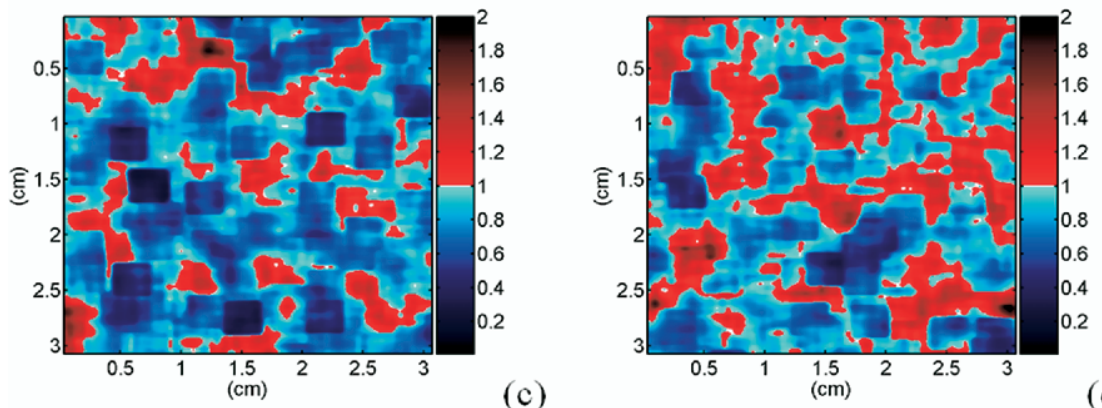

(d)

Fig. 5. The Nakagami parametric images with a pseudocolor display for the type-I phantoms of various scatterer concentrations. (a) 2 scatterers $/ \mathrm{mm}^{2}$; (b) 4 scatterers $/ \mathrm{mm}^{2}$; (c) 8 scatterers $/ \mathrm{mm}^{2}$; (d) 16 scatterers $/ \mathrm{mm}^{2}$.

ness of the B-mode image but not altering the image contrast. Hence, the brightness and the contrast of the B-mode image strongly depend on the dynamic range and the system gain, implying that the B-mode images measured by different diagnosticians using different ultrasonic systems for the same examined tissue may be different. Figure 2 shows the B-mode images and the gray-scale histograms for the phantoms with the scatterer
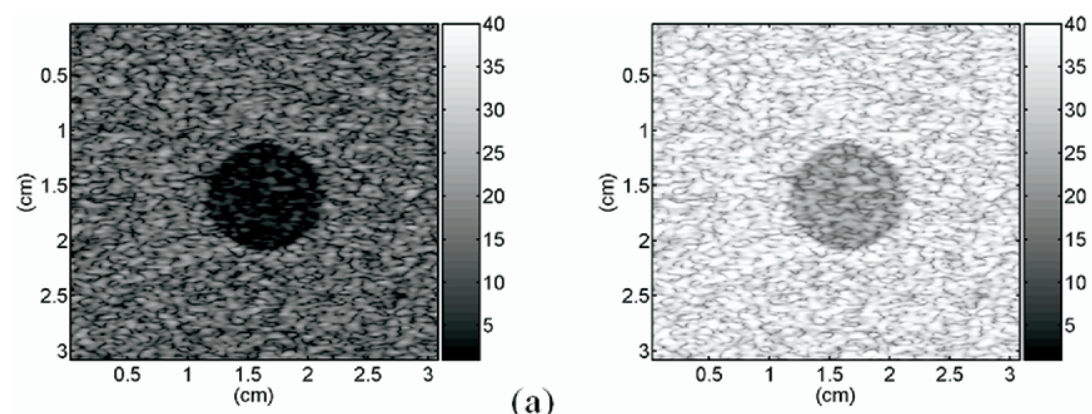

(b)
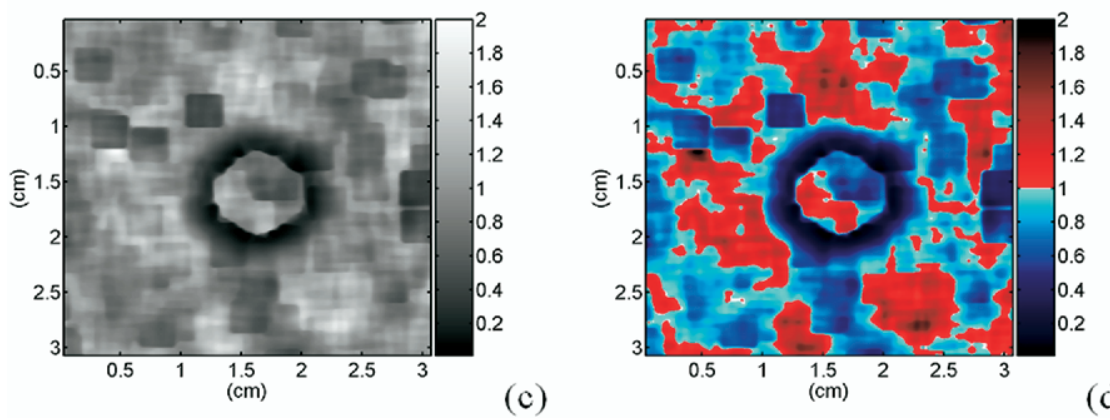

Fig. 6. The B-mode and Nakagami images of the type-II phantom (no. 1). (a) and (b) The B-mode images using gains of -10 and $10 \mathrm{~dB}$; (c) and (d) The Nakagami images using gray-scale and pseudocolor. 

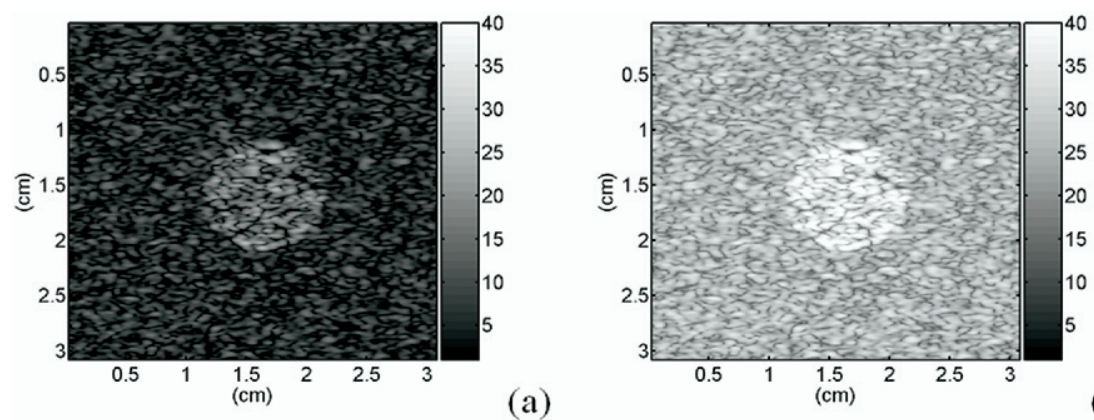

(b)
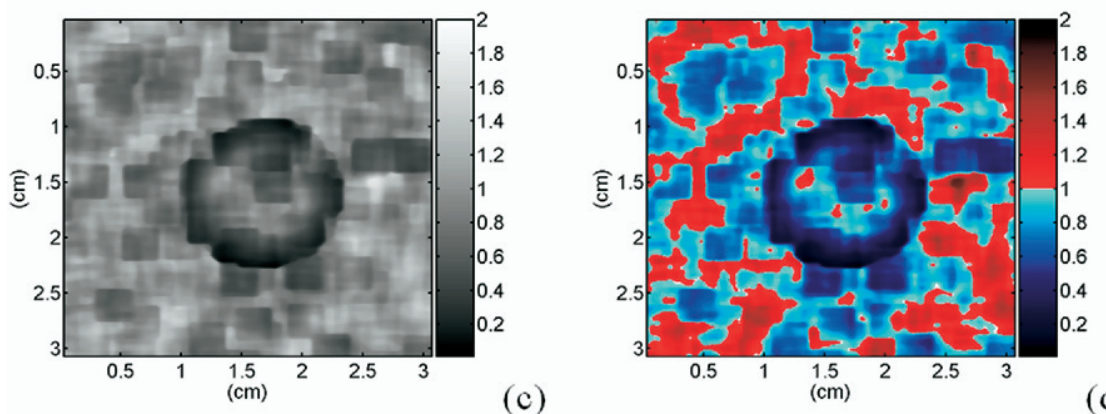

Fig. 7. The B-mode and Nakagami images of the type-II phantom (no. 2). (a) and (b) The B-mode images using gains of -10 and $10 \mathrm{~dB}$; (c) and (d) The Nakagami images using gray-scale and pseudocolor.

concentrations of 16 scatterers $/ \mathrm{mm}^{2}$, indicating that the B-mode images are not consistent when different operation settings are used. Moreover, comparison of Fig. 1 with Fig. 2 shows that the B-mode images with various scatterer concentrations would become similar to each other under certain dynamic ranges and gains, resulting in the fact that the scatterer properties in a biological tissue cannot be identified in the conventional gray-scale
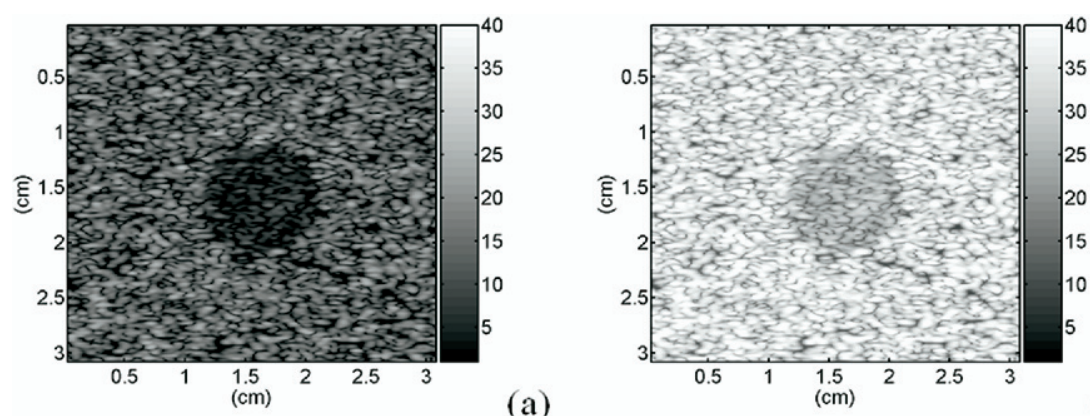

(a)

(b)
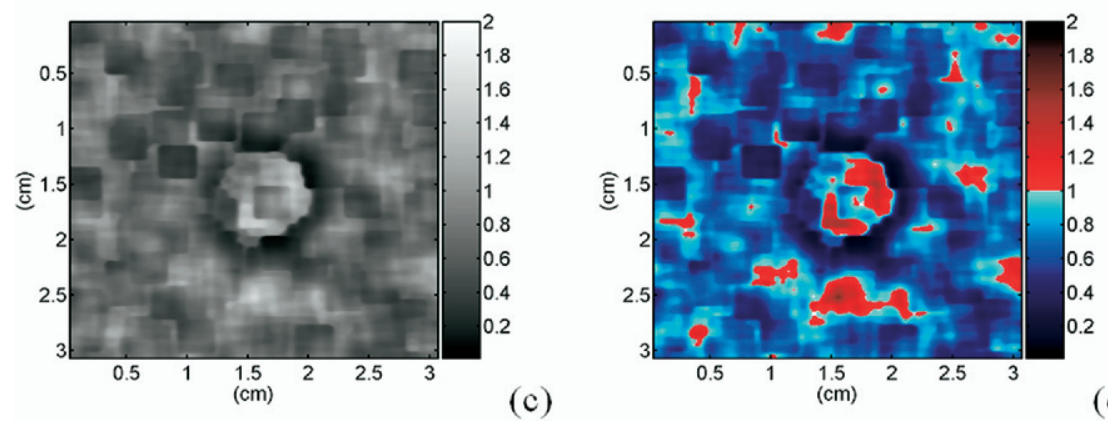

(d)

Fig. 8. The B-mode and Nakagami images of the type-II phantom (no. 3). (a) and (b) The B-mode images using gains of -10 and $10 \mathrm{~dB}$; (c) and (d) The Nakagami images using gray-scale and pseudocolor. 
Table 2. Comparison of the Nakagami parametric image with the conventional B-mode image

\begin{tabular}{|c|c|c|}
\hline & B-mode image & Nakagami image \\
\hline Image pixel & Grayscale & $\begin{array}{l}\text { Local Nakagami } \\
\text { parameter }\end{array}$ \\
\hline $\begin{array}{l}\text { Image physical } \\
\text { meaning }\end{array}$ & $\begin{array}{l}\text { Relative echo } \\
\text { intensity }\end{array}$ & Envelope statistics \\
\hline Image type & Qualitative & Quantitative \\
\hline Resolution & Relatively better & $\begin{array}{l}\text { Relatively poor } \\
\text { Scatterer concentration }\end{array}$ \\
\hline Medical application & Morphology analysis & differentiation \\
\hline
\end{tabular}

image. These results and previous studies (Gosink et al. 1979; Walach et al. 1989; Shung and Thieme 1993; Raju and Srinivasan 2002; Raju et al. 2003) demonstrate that the tissue properties are difficult to obtain from the B-scans.

The optimal window size used to form the Nakagami image

To visualize the scatterer properties of the phantom, the Nakagami parametric images of different scatterer concentrations are constructed. Before this, the optimal size of the sliding window used to form the Nakagami parametric image has to be determined. Figure 3 shows the mean parameter $\bar{m}$ estimated from the envelope signal of the image scan-line and the average $\bar{m}_{w}$ of the parameter $m_{w}$ estimated from the local envelope data in different sizes of the window. The parameter $\bar{m}$ is increased from 0.41 to 0.91 with increasing scatterer concentration from 2 to 16 scatterers $/ \mathrm{mm}^{2}$. This result agrees well with those in the previous studies (Shankar 2000; Tsui and Wang 2004; Tsui et al. 2005a, 2005b), indicating that the global pdf of the backscattered envelope would change from the pre-Rayleigh to Rayleigh statistics with increasing the scatterer concentration. Note that, with increasing window size, the curve of $\bar{m}_{w}$ as the scatterer concentration gradually approaches that of $\bar{m}$. It is found that the parameter $\bar{m}_{w}$ is increased from about 0.42 to 0.93 with increasing scatterer concentration from 2 to 16 scatterers $/ \mathrm{mm}^{2}$ when the window size is $2.7 \times$ $2.7 \mathrm{~mm}^{2}$. This demonstrates that an appropriate size of the window can satisfy the stable estimation of the parameter $m_{w}$, enabling the average of the local Nakagami parameters $\bar{m}_{w}$ to represent the correct global backscattered statistics, as assumed in the section on Methods. More precisely, this result further implies that the size of the window corresponding to the square with the side length equal to three times the pulse length may be an optimal size for the stable estimation of $m_{w}$ (because 2.7 $\mathrm{mm}$ is about three times the pulse length). On the other hand, the window size cannot be too large, as we could take $m_{w}$ to distinguish the variation of the local scatterer concentration. Accordingly, the window size of $2.7 \times$ $2.7 \mathrm{~mm}^{2}$ is applied in the following constructions of the Nakagami image.

\section{The Nakagami images of the type-I phantom}

Figure 4 shows the gray-scale Nakagami images of the type-I phantoms with different scatterer concentrations ranging from 2 to $16 \mathrm{scatterers} / \mathrm{mm}^{2}$. The overall brightness of the Nakagami image is increased with increasing the scatterer concentration from 2 to 16 scatterers $/ \mathrm{mm}^{2}$, because the global backscattered statistics varied from the pre-Rayleigh to Rayleigh distribution with increasing scatterer concentration, making $\bar{m}_{w}$ increase. However, the brightness in each local region in the Nakagami image is different for each phantom, due to the fact that each local region has different values of $m_{w}$. Namely, we have formed the Nakagami image with $m_{w}$. The Nakagami image can be applied to the detection of the local scatterer aggregation in a tissue. Furthermore, compared with the B-mode image, the Nakagami image is relatively robust in producing a consistent image for different users, imaging systems and operation settings. The reason for the consistent image lies in the fact that the pixel of the Nakagami image is $m_{w}$, which is only dependent on the shape of the local envelope statistics and not affected by the magnitude of the local envelope signal. Figure 5 shows the pseudocolor Nakagami images of the four type-I phantoms, depicting that the pseudocolor endows the Nakagami image with a more direct and convenient way to visualize the scatterer properties of the biological tissues. It is found that the Nakagami images of the phantoms with relatively low scatterer concentrations have more blue shades and less red regions. This represents that the low scatterer concentration would cause a larger spacing between the scatterers in a medium, rendering most statistics of the local backscattered envelope to be the pre-Rayleigh distributions. On the other hand, the red region spread with increasing scatterer concentration. It means that high scatterer concentration results in a comparatively small spacing between the scatterers in a medium, leading to a conspicuous effect of the scatterer aggregation, and that the most of the local envelope signals tend to be the post-Rayleigh distribution.

\section{The B-mode and Nakagami-mode images of the type-II phantom}

To further evaluate the performance of the Nakagami parametric image in characterizing biological tissues, three type-II phantoms are simulated to compare their B-mode and Nakagami images. Figure 6 shows the B-scans and Nakagami images of the phantom no. 1. Due to the scatterer echogenicity coefficient in the background being fivefold of that in the cylinder, the scatter- 
ers in the cylinder are relatively weak scatterers, leading to the weak backscattered signals and darker brightness of the B-mode image. Note that the cylinder in the B-mode image behaves as an area without any returned echoes when the system gain is low. This may easily mislead the diagnosticians with less experience in describing the properties of the scatterers in the cylinder. Instead, the Nakagami image clearly indicates that the cylinder is not an empty region. Figure 7 shows the B-mode and Nakagami-mode images of the phantom no. 2. The scatterer properties for the phantom no. 2 enable the B-mode image of the cylinder region to be brighter. This may produce a problem that the diagnosticians would treat the cylinder object as a high scatterer concentration area. In the meantime, the Nakagami image indicates that the scatterer concentration of the cylinder is actually lower than the background. Figure 8 shows the B-mode and Nakagami images of the phantom no. 3. The scatterer properties of the phantom no. 3 make the B-mode image in the cylinder darker. As a result, we cannot tell that the scatterer concentration of the cylinder is higher than that of the background using the B-mode image. On the contrary, the Nakagami image can successfully indicate that the cylinder region has a higher scatterer concentration than the background. In summary, the comparisons of the Nakagami image with the conventional B-scans are listed in Table 2. We consider that the B-mode image and the Nakagami image are complementary to each other. The B-mode image is responsible for the morphology analysis and the Nakagami image is used to evaluate the distribution of the scatterers in the tissue.

\section{CONCLUDING REMARKS}

In this study, we have explored the ultrasonic parametric image based on the map of the Nakagami parameter. In particular, computer simulations have been carried out to generate phantoms of various scatterer properties for comparing the B-mode and Nakagami-mode images in order to evaluate their individual performances in the ultrasonic tissue characterization. According to the simulated results, the optimal size of the sliding window used to construct the Nakagami image is the square with the side length equal to three times the pulse length of the incident ultrasound. The Nakagami image constructed using this window size can provide both the global and local backscattered statistics of the ultrasonic signals in a tissue, demonstrating that the Nakagami image has an outstanding ability to detect the local scatterer concentrations in a tissue. Moreover, compared with the Bmode image that is easily influenced by the factors related to the system and operator, the Nakagami image yields a comparatively consistent image result for differ- ent dynamic ranges and system gains. This is because the Nakagami image formation is based on the shape of the local backscattered envelope in the tissue and would not be affected by the magnitude of the ultrasonic backscattered signal. Furthermore, this imaging principle associated with the Nakagami image enables extraction of the weak backscattering information that may be lost in the B-mode image. For these reasons, the Nakagami image has a great potential to become a new diagnostic tool that is easily combined with the use of the conventional B-scans simultaneously to visualize the structures and the scatterer properties in the tissue. Nevertheless, experimental and clinical validations must be carried out before the local scatterer concentration image based on the Nakagami model can be used as a reliable clinical or research tool.

\section{REFERENCES}

Bamber JC, Dickinson RJ. Ultrasonic B-scanning: A computer simulation. Phys Med Biol 1980;25:463-479.

Bamber JC, Hill CR. Acoustic properties of normal and cancerous human liver-I dependence on pathological condition. Ultrasound Med Biol 1981;7:121-133.

Bamber JC, Hill CR, King JA. Acoustic properties of normal and cancerous human liver-II dependence on tissue structure. Ultrasound Med Biol 1981;7:135-144.

Berson M, Roncin A, Pourcelot L. Compound scanning with an electrically steered beam. Ultrason Imag 1981;3:303-308.

Burckhardt CB. Speckle in ultrasound B-mode scans. IEEE Trans Sonics Ultrason 1978;SU-25:1-6.

Cloutier G, Daronatand M, Savery D, Garcia D, Durand LG, Foster FS. Non-Gaussian statistics and temporal variations of the ultrasound signal backscattered by blood at frequencies between 10 and 58 MHz. J Acoust Soc Am 2004;116:566-577.

Davignon F, Deprez JF, Basset O. A parametric imaging approach for the segmentation of ultrasound data. Ultrasonics 2005;43:789-801.

Dutt V, Greenleaf JF. Ultrasound echo envelope analysis using a homodyned K distribution signal model. Ultrason Imag 1994;16: 265-287.

Fontaine I, Bertrand M, Cloutier G. A system-based approach to modeling the ultrasound signal backscattered by red blood cells Biophysical J 1999;77:2387-2399.

Fontaine I, Savery D, Cloutier G. Simulation of ultrasound backscattering by red cell aggregates: Effect of shear rate and anisotropy. Biophysical J 2002;82:1696-1710.

Gosink BB, Lemon SK, Scheible W, Leopold GR. Accuracy of ultrasonography in diagnosis of hepatocellular disease. Am J Roentgenol 1979;133:19-23.

Hampshire JB II, Strohbehn JW, McDaniel MD, Waugh JL, James DH. Probability density of myocardial ultrasonic backscatter. Proceedings of the 14th Annual Northeast Bioeng Conference. Durham, NH: IEEE. 1988:305-308.

Insana MF, Wagner RF, Brown DG, Hall TJ. Describing small-scale structure in random media using pulse-echo ultrasound. J Acoust Soc Am 1990;87:179-192.

Karmeshu, Agrawal R. Study of ultrasonic echo envelope based on Nakagami-inverse Gaussian distribution. Ultrasound Med Biol 2006;32:371-376.

Koláŕ R, Jiřik R, Jan J. Estimator comparison of the Nakagami-m parameter and its application in echocardiography. Radioengineering 2004;13:8-12.

Meunier J, Bertrand M. Echogrpahic image mean gray level changes with tissue dynamics: A system-based model study. IEEE Trans Biomed Eng 1995;42:403-410. 
Raju BI, Srinivasan MA. Statistics of envelope of high-frequency ultrasonic backscatter from human skin in vivo. IEEE Trans Ultrason Ferroelec Freq Contr 2002;49:871-882.

Raju BI, Swindells KJ, Gonzalez S, Srinivasan MA. Quantitative ultrasonic methods for characterization of skin lesions in vivo. Ultrasound Med Biol 2003;29:825-838.

Shankar PM. A model for ultrasonic scattering from tissues based on K-distribution. Phys Med Biol 1995;40:1633-1649.

Shankar PM. A general statistical model for ultrasonic backscattering from tissues. IEEE Trans Ultrason Ferroelec Freq Contr 2000;47: 727-736.

Shankar PM, Dumane VA, Reid JM, et al. Classification of ultrasonic B-mode images of breast masses using Nakagami distribution. IEEE Trans Ultrason Ferroelec Freq Contr 2001;48:569-580.

Shankar PM. Statistical modeling of scattering from biological media. J Acoust Soc Am 2002;111:2463.

Shankar PM. A compounding scattering pdf for the ultrasonic echo envelope and its relationship to $\mathrm{K}$ and Nakagami distributions. IEEE Trans Ultrason Ferroelec Freq Contr 2003a;50:339-343.

Shankar PM, Dumane VA, George T, et al. Classification of breast masses in ultrasonic B scans using Nakagami and $\mathrm{K}$ distributions. Phys Med Biol 2003b;48:2229-2240.

Shankar PM. The use of the compounding probability density function in ultrasonic tissue characterization. Phys Med Biol 2004;49:1007-1015.

Shung KK, Smith MB, Tsui B. Principles of medical imaging. San Diego: Academic Press, 1992.

Shung KK, Thieme GA. Ultrasonic scattering in biological tissues. Boca Raton: CRC Press, 1993

Teh BG, Cloutier G. Modeling and analysis of ultrasound backscattering by spherical aggregates and rouleaux of red blood cell. IEEE Trans Ultrason Ferroelec Freq Contr 2000;47:1025-1035.

Thijssen JM. Ultrasonic tissue characterization and echographic imaging. Phys Med Biol 1989;34:1667-1674.
Trahey GE, Allison JW, Smith SW, von Ramm OT. A quantitative approach to speckle reduction via frequency compounding. Ultrason Imag 1986;8:151-164.

Tsui PH, Wang SH. The effect of the transducer characteristics on the estimation of Nakagami parameter as a function of scatterer concentration. Ultrasound Med Biol 2004;30:1345-1353.

Tsui PH, Wang SH, Huang CC. The effect of logarithmic compression on estimation of Nakagami parameter for ultrasonic tissue characterization. Phys Med Biol 2005a;50:3235-3244.

Tsui PH, Wang SH, Huang CC, Chiu CY. Quantitative analysis of noise influence on the detection of scatterer concentration by $\mathrm{Na}$ kagami parameter. J Med Biol Eng 2005b;25:45-51.

Valckx FMJ, Thijssen JM, van Geemen AJ, Rotteveel JJ, Mullaart R. Calibrated parametric medical ultrasound imaging. Ultrason Imag 2000;22:57-72.

Wachowiak MP, Smolikova R, Tourassi GD, Elmaghraby AS. General ultrasound speckle models in determining scatterer density. P SPIE 2002;4687:285-295.

Wagner RF, Insana MF, Brown DG. Statistical properties of radiofrequency and envelope detected signals with applications to medical ultrasound. J Opt Soc Am 1987;4:910-922.

Walach E, Shmulewitz A, Itzchak Y, Hetman Z. Local tissue attenuation images based on pulsed-echo ultrasound scans. IEEE Trans Biomed Eng 1989;36:211-221.

Wang SH, Tasi FC. Characterization of bony tissues from ultrasonic backscattering using statistical models. IEEE Ultrason Symp Proc 2001;2:1205-1208.

Weng L, Reid JM, Shankar PM, Soetanto K. Ultrasound speckle analysis based on the K distribution. J Acoust Soc Am 1991;89: 2992-2995.

Zimmer Y, Akselrod S, Tepper R. The distribution of the local entropy in ultrasound images. Ultrasound Med Biol 1996;22:431-439. 